\title{
Heat energy as measure of matter chaotization
}

\author{
Vitaly Pavlovich Malyshev, Asrta Mundukovna Makasheva, Yana Alekseevna Fedorovich
}

Chemical and metallurgical institute. Karaganda, Republic of Kazakhstan

Email address:

eia_hmi@mail.ru (V. P. Malyshev), astra_mun@mail.ru (A. M. Makasheva), yana0071993@mail.ru (Y. A. Fedorovich)

\section{To cite this article:}

Vitaly Pavlovich Malyshev, Asrta Mundukovna Makasheva, Yana Alekseevna Fedorovich. Heat Energy as Measure of Matter Chaotization. American Journal of Physical Chemistry. Vol. 3, No. 5, 2014, pp. 72-76. doi: 10.11648/j.ajpc.20140305.14

\begin{abstract}
The heat energy, which is a measure of chaotic motion of particles and which is determined by Boltzmann distribution that is valid not only for gaseous but condensed state of matter, could be an analytical tool to reveal the united nature of solid, liquid and gaseous states based on introduction of new concepts of virtual crystal-mobile, liquid-mobile and vapor-mobile particles.
\end{abstract}

Keywords: Heat Energy, Randomized Particles, Barriers of Melting and Boiling

\section{Introduction}

It is well know that such main states of matter like solid, liquid and gas differ by the rate of particles chaotization. State transition is accompanied by overcoming of energy barrier. The temperature at melting point and boiling point is unchanged, and the system has $R T_{m}$ and $R T_{b}$ heat energy correspondingly at these points for both balanced phases.

In general, possession of RT level heat energy at any temperature is founded by Boltzmann distribution (of energy spectrum); it is an average integral quantity of this distribution. Heating energy is distributed by particles at any temperature and in any state in the range from $\varepsilon=0$ to $\varepsilon \rightarrow \infty$. That is why there is a certain amount of particles which are able or unable to overcome some barrier, for instance, the barrier of melting or boiling, i.e. $R T_{m}$ or $R T_{b}$.

\section{Accounting Barriers of Chaotization}

These amounts could be calculated based on the general formula which also could be developed from Boltzmann distribution to any given activation barrier $E_{a}(\mathrm{~J} / \mathrm{mol})$ and for the amount of overbarrier particles which is equal to

$$
P_{>}=\exp \left[-E_{a} /(R T)\right] \text {. }
$$

Correspondingly, this formula for the amount of underbarrier particles is as follows

$$
P_{<}=1-\exp \left[-E_{a} /(R T)\right],
$$

because the total sum of all particles' ratios is strictly equal to one:

$$
\sum_{i=1}^{m} p_{i}=1
$$

where $P_{i}$ is the amount of particles with $\varepsilon_{i}$ energy (or $E_{i}=$ $N_{A} \varepsilon_{I}$ in mol) and it is subordinated by Boltzmann distribution

$$
P_{i}=N_{i} / N=\exp \left[-\varepsilon_{i} /(k T)\right] / \sum_{i=1}^{m} \exp \left[-\varepsilon_{i} /(k T)\right] .
$$

$N_{i}$ is a number of particles with medium energy $\varepsilon_{i} ;, N$ is a total number of particles, $k$ is Boltzmann constant and $m$ is a number of calculated energy levels. $P_{i}$ could be regarded as probability to reveal particles with $\varepsilon_{i}$ energy as well.

Based on equations (1) and (2), the rate of overbarrier particles in the temperature range from 0 to $T \rightarrow \infty$ will be increased from 0 to 1 ; and the rate of underbarrier particles will be decreased from 1 to 0 .

What is the ratio of both particle types in melting and boiling points?

Thus, taking into account the heating barrier of melting temperature $R T_{m}$ the equations (1) and (2) will be as follows

$$
\begin{gathered}
P_{>}=\exp \left[-R T_{m} /(R T)\right]=\exp \left(-T_{m} / T\right), \\
P_{<}=1-\exp \left[-T_{m} / T\right] .
\end{gathered}
$$

At the melting point these rates will be as follows correspondingly:

$$
P_{m>}=\exp \left(-T_{m} / T\right)=\exp (-1) \cong 0,37,
$$




$$
P_{m<}=1-\exp (-1) \cong 0,63,
$$

Thus the major rate of the particles will be underbarrier particles and the minor part will be the overbarrier ones. This fact suggests that there is some critical rate of particles which is able to hold the matter in the long-range order, i.e. as a crystal. This critical rate is constituted by the particles which stay in the sites because their insufficient energy. Other overbarrier particles are able to transfer into inter-site position and create virtual spot defects and weaken the links of the matter in general, which is still solid.

In other words, the most defective equilibrium lattice is formed at the melting point. In this lattice every elementary cell has the ratio of sites occupied by particles to vacant sites which is numerically described as 0,63:0,37.

There are several ways to interpret this ratio. Firstly, there is necessity to have the mere majority of supporting and structure-forming particles of the lattice towards the destructing particles. Secondly, it is close to the golden ratio of $\sim 0,62: 0,38$, which is common for the most balanced link of the structure-forming component and the additional chaotic adjustable component of the wide range of systems; this link is proved by Shannon entropy [1,2]. Thirdly, it could be interpreted based on the geometrical ideas of preserving the three-dimensional configuration and linkage of the lattice. Based on the example of the simplest cubic lattice with eight sites, it is obvious that such preservation is possible if the number of the particles in the lattice is higher than four (only for one side), i.e. more than five; in this case the ratio of the sites with particles in the softest crystal lattice is $5 / 8=0,625$, that corresponds to the proportion of the golden section and proportion of sub-barrier particles (8).

\section{Using of Chaotization Barriers for New Treatment of Solid, Liquid and Gaseous States of Matter}

New understanding of melting in general corresponds to the solid-state theory of Einstein. According to the theory, the lattice destruction occurs when the average amplitude of particles oscillation is equal to the average distance between them. Working with average values presupposes the coordination of the amplitude of oscillation and distance between particles with their distribution, according to which there are particles with the higher average amplitude and the distance between them is higher average at any temperature. The virtual presence of the particles with higher average amplitudes and higher average distances between the sites of the crystal lattice are determined by the oscillatory mode of motion. To describe this process the more general characteristic is used: it is kinetic energy of the chaotic movements of the particles. That is why such approach could be regarded as the approach which corresponds to the complementary principle. This approach allows quantitative evaluation of a crystal lattice at the melting point by the ratio of the virtual vacant sites and the virtual occupied sites because the underbarrier particles are also virtual due to their participation in exchanging encounters.

The lattice virtuality could be evaluated at this state. Thus, if 5 sites out of 8 sites of a cubic lattice should be occupied, this could be implemented by the non-repeating number of combinations from 8 to 5 .

$$
C_{8}^{5}=\frac{8 !}{5 !(8-5) !}=56 \text {. }
$$

Specifically, the softest cubic lattice could form this number of configurations while remaining integral and virtual at the same time and preserving the ability to reflect radiation by virtually stable sides. Thus the whole crystal is virtual but clearly seen like a rainbow in the sky. Still free sites should be regarded in the same way, i.e. as virtually vacant. In this case they are dynamically unstable and coordinated by balanced exchange Boltzmann distribution.

According to the stated above, the ratio of underbarrier particles in the range from 0 to $T_{m}$ to overcome $R T_{m}$ barrier changes from 1 to $\sim 0,63$ while being the highest one in comparison with the overbarrier particles ratio which is from 0 to 0,37 in this range. Taking into account the function of underbarrier particles to preserve the crystal state and their virtuality these particles were named crystal-mobile and their ratio is indicated by $\mathrm{crm}$ index in the framework of general conception of chaotic particles [3-7]:

$$
P_{c r m}=1-\exp \left[-T_{m} / T\right] \text {. }
$$

Taking into account the matter transition from solid state to liquid and gaseous ones, the overbarrier particles ratio should be differentiated according to overcoming and nonovercoming of heat barrier of boiling. So, the overbarrier particles ratio according to $R T_{b}$ value is

$$
P_{>}=\exp \left[-R T_{b} /(R T)\right]=\exp \left(-T_{b} / T\right)=P_{v m} .
$$

It describes their number at any temperature at any state of matter because according to Boltzmann distribution the highest-energy particles are able to be created at any temperature except absolute zero of temperature. These particles do not participate in long-range and short-range links; they virtually leave the crystal lattice sites for interstitial lattice sites and form equilibrium vapor above solid and liquid matter. This fact confirms the existence of such particles. Such particles are named vapor-mobile and their ratio is indicated by $P_{v m}$.

Along with crystal-mobile and vapor-mobile particles there should be such particles which energy is higher than the melting barrier but less than the boiling barrier; and according to its energy status these articles should be responsible for the short-range quasi-structural links (not long-range) which are typical for liquids. That is why they are named liquid-mobile and their ratio is determined by the difference between one and ratios of $P_{c r m}(9)$ and $P_{v m}(10)$ :

$$
P_{l q m}=1-P_{c r m}-P_{v m}=\exp \left(-T_{m} / T\right)-\exp \left(-T_{b} / T\right)
$$


General understanding of Boltzmann distribution (4) at melting temperature and boiling temperature by energy rates (in mol) is shown in Figure 1. Temperature dependences of the ratio of the chaotic particles of three energy classes are shown in Figure 2 (illustrated by barium with $T_{m}=1000 \mathrm{~K}$, and $\left.T_{b}=2170 \mathrm{~K}\right)$.

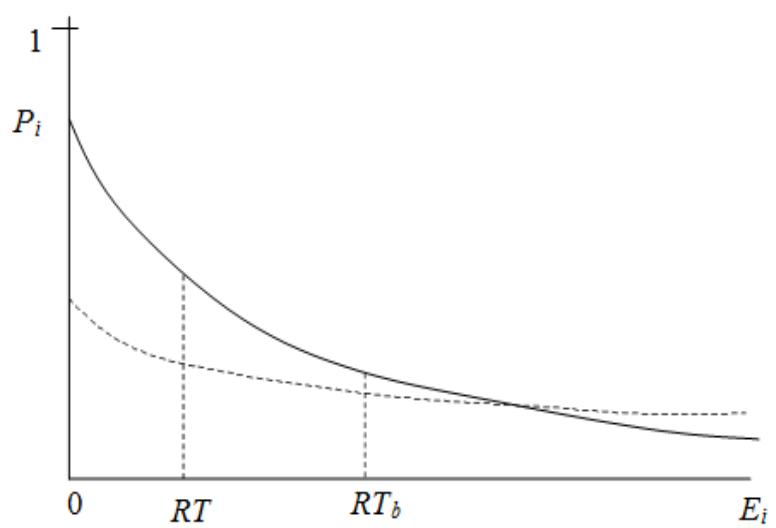

Figure 1. Boltzmann Distribution (Energy Spectrum) at Boiling and Melting

$P_{i}$ is ratio of particles with $E_{i}$ energy (in mol); $E_{i}$ - average heating energy of particle at i-level of energy. Full line is for $T_{m}$, dashed line is for $T_{b}$. $R_{m}$ and $\mathrm{RT}_{\mathrm{b}}$ are energy barriers at melting temperature and boiling temperature

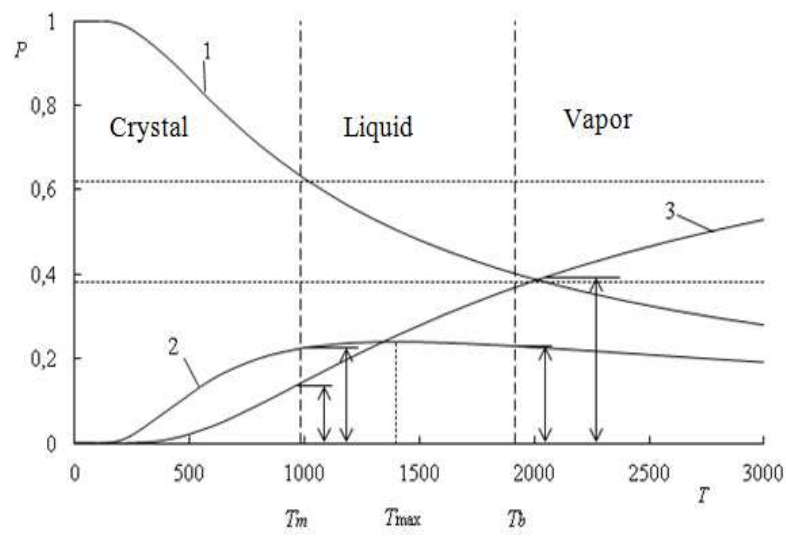

Figure 2. Dependency of Chaotic Particles Ratio on Temperature for Barium.

$\mathrm{P}$ is ratio of chaotic particles; $\mathrm{T}$ is temperature. Temperature dependences of the ratio of 1 - crystal-mobile, 2 - liquid-mobile and 3- vapor-mobile particles. Horizontal lines are for the values of golden ratio $(\sim 0,62$ and $\sim$ $0,38)$, vertical lines are for melting point and boiling point. Arrows denote the liquid-mobile particles ration and vapor-mobile particles ration which are in the golden ratio. Temperature of liquid-mobile particles maximum is highlighted.

Liquid-mobile particles along with other types of particles should be presented in every state and should participate in virtual exchanging processes. But if the virtuality of crystalmobile particles does not obstacle the preservation and perception (through sight and tactile sense) of the crystal lattice integrity and the virtuality of vapor-mobile particles does not obstacle the formation and experimental registration of crystal equilibrium vapor (through olfaction and sight), so how the virtuality of liquid-mobile particle presented in solid matter is revealed?
Unfortunately, such particles are not directly perceived in solid matter. Probably, that is why the unity of three states of matter at every of them was not obvious and was not specially analyzed. Nevertheless, such properties of solid matter could be easily explained by the presence of liquidmobile particles [8].

First of all, there is plasticity. It increases along with temperature growth, at that the ration of liquid-mobile particles is growing as well. The matter becomes liquid not "at once" but due to gradual increase of liquid-mobile particles number till the critical ratio with crystal-mobile particles. Thus the quantity of heat energy $R T$ at any temperature is as lower as it differs from the barrier energy $R T_{m}$ at melting point. Additional energy is needed to achieve this barrier, for example, mechanical energy of plastic deformation in the following amount:

$$
E_{m e c}=R T_{m}-R T=R\left(T_{m}-T\right) .
$$

Because this statement is based on the balanced distribution of heat energy, it characterizes minimal necessary and useful energy to transfer matter to liquid state. That is why formula (12) was used for the first time to calculate the unknown energy coefficient of efficiency of plastic deformation, taking into account its completeness illustrated by the example of a nine-cell rolling mill work to produce a copper wire rod [8] and comparing the calculated and actual expenditures of energy. Thus the value of the energy efficiency coefficient is quite realistic and it is differentiated by roughening cells and finishing cells according to operations. This factor also determines improving the forging property of metals at higher temperatures.

Such understanding of plasticity independently explains creep which is a property of solid matter to behave as liquid under the influence of even small but constant load. Creep deformation results in moving of glaciers and gradual deforming of metal structures.

The overbarrier virtual micro-particle could not be registered in the crystal and they are detected by creating interferences, e.g. by lower intensity and increased width of spectrum lines, and they serve as an energy storage for forming registered defects at kinetic conditions which could be expressed by the reference of activation energy of selfdiffusion determined by an experiment to the range between heat barriers of melting and boiling, i.e. the area of energy implementation of liquid-mobile particles [9].

In general, there could be drawn a following conclusion based the application of chaotic particles conception to solid matter: there is a definite correlation between the ratio of crystal-mobile particles and the preservation of the longrange linkage in a virtual crystal lattice up to destruction of this link at melting point, and the responsibility of crystalmobile particles for solidity and fragility of matter which is based by direct comparison of the ratio of these particles with fragility at various temperatures [10]. Actually, especially crystal-mobile particles allow solidity of solid state of matter. In turn, the ratio of liquid-mobile particles, which provide 
plasticity, could be the measure of liquid properties of solid matter. Vapor-mobile particles are the most energetic particles in solid matter which are balanced with the similar ratio in gas above solid matter. Based on this fact the conclusion of virtual gaseous phase in solid matter could be made.

More detailed understanding of liquid state from the point of chaotic particles concept is given in our monograph [11]. It proves that viscosity and density of liquid and their dependency on temperature are defined by the ratio of crystal-mobile particles which form clusters and associates that make virtual solid phase of liquid state. Fluidity, which is not the inverse value of viscosity but the opposite value, is directly related to the ratio of single and not-linked with clusters particles, which are liquid-mobile and vapor-mobile. This fact gives new understanding of liquid characteristics. In turns, the temperature dependency of liquid density is directly connected with the ratio of crystal-mobile particles which form more compact clusters and associates. There is an example to analyze the application of the chaotic particles conception to analytical description of the liquid lithium viscosity temperature dependency. According to the monograph [11] the crystal mobile particles (9) are a reservoir for the quasicrystalline virtual (and dynamically unstable) clusters formation, which, correspondingly, are a reservoir for the formation of larger associates. Such hierarchy of virtual solid state structural forms in liquid are also implemented by the hierarchic cluster and associate model of the dynamic viscosity temperature dependency:

$$
\eta=\eta_{1}\left(T_{1} / T\right)^{a=a_{2}\left(T_{2} / T\right) b}
$$

where the lower base of the $\left(T_{1} / T\right)$ power corresponds the dynamic of the clusters' destruction and the higher base fo the $\left(T_{2} / T\right)$ power corresponds the clusters' associate destruction while $a$ is the clusters' association rate, i.e. the average number of clusters in a associate; $b$ is the clusters' aggregation rate, i.e. the average number of associates in an aggregate. Thus the temperature dependency of viscosity is directly connected with the reduction of the rate of clusters and associates which, while being multi-particles structures, define the liquid consistence and viscosity.

To adopt this model with experimental data, three check points $T_{1}, \eta_{1}, T_{2}, \eta_{2}$ and $T_{3}, \eta_{3}$, are to be applied to find the values.

$$
\begin{gathered}
a_{2}=\frac{\ln \left(\eta_{2} / \eta_{1}\right)}{\ln \left(T_{1} / T_{2}\right)}, \\
a_{3}=\frac{\ln \left(\eta_{3} / \eta_{1}\right)}{\ln \left(T_{1} / T_{3}\right)}, \\
b=\frac{\ln \left(a_{3} / a_{2}\right)}{\ln \left(T_{2} / T_{3}\right)} .
\end{gathered}
$$

The points are selected on a random basis but it is recommended to represent evenly the whole massive of the experimental data (from the beginning, middle and end of the massive). In general such approach proved its high efficiency which was demonstrated on the large group of elementary substances [11] and later on the complicated substances [12].

There are some reference data of lithium dynamic viscosity in the wide range of temperatures [13]. Three check points $T_{1}=523 \mathrm{~K}, \quad \eta_{1}=0,503 \mathrm{mPa} \cdot \mathrm{s}, \quad T_{2}=1073 \mathrm{~K}$, $\eta_{2}=0,208 \mathrm{mPa} \cdot \mathrm{s}$ and $T_{3}=1923 \mathrm{~K}, \eta_{3}=0,145 \mathrm{mPa} \cdot \mathrm{s}$ were chosen. Based on these data and according to the formulae (13) - (16) the cluster and associate model of lithium dynamic viscosity was identified:

$$
\eta=0,503(523 / \mathrm{T})^{1,0413\left(\frac{1073}{\mathrm{~T}}\right)^{0,1478}},
$$

\begin{tabular}{|c|c|c|c|c|c|c|c|}
\hline$T, \mathbf{K}$ & $\eta[13], \mathrm{mPa} \cdot \mathrm{s}$ & $\eta(17), \mathrm{mPa} \cdot \mathrm{s}$ & $a$ & $T, \mathbf{K}$ & $\eta[13], \mathrm{mPa} \cdot \mathrm{s}$ & $\eta(17), \mathrm{mPa} \cdot \mathrm{s}$ & $a$ \\
\hline$T_{m}=453,7$ & - & 0,595 & 1,183 & 1423 & 0,185 & 0,185 & 0,999 \\
\hline 473 & 0,566 & 0,566 & 1,175 & 1473 & 0,180 & 0,180 & 0,994 \\
\hline 523 & 0,503 & 0,503 & 1,160 & 1523 & 0,175 & 0,175 & 0,989 \\
\hline 573 & 0,453 & 0,453 & 1,143 & 1573 & 0,170 & 0,170 & 0,984 \\
\hline 623 & 0,412 & 0,413 & 1,128 & $T_{b}=1615$ & - & 0,167 & 0,980 \\
\hline 673 & 0,379 & 0,380 & 1,116 & 1623 & 0,166 & 0,166 & 0,980 \\
\hline 723 & 0,352 & 0,352 & 1,110 & 1673 & 0,162 & 0,162 & 0,975 \\
\hline 773 & 0,328 & 0,328 & 1,093 & 1723 & 0,158 & 0,158 & 0,971 \\
\hline 823 & 0,308 & 0,308 & 1,083 & 1773 & 0,155 & 0,155 & 0,967 \\
\hline 873 & 0,290 & 0,290 & 1,074 & 1823 & 0,151 & 0,151 & 0,963 \\
\hline 923 & 0,275 & 0,275 & 1,065 & 1873 & 0,148 & 0,148 & 0,959 \\
\hline 973 & 0,261 & 0,261 & 1,057 & 1923 & 0,145 & 0,145 & 0,955 \\
\hline 1023 & 0,249 & 0,249 & 1,049 & 1973 & 0,142 & 0,142 & 0,952 \\
\hline 1073 & 0,238 & 0,238 & 1,041 & 2023 & 0,139 & 0,140 & 0,948 \\
\hline 1123 & 0,228 & 0,228 & 1,034 & 2073 & 0,137 & 0,137 & 0,945 \\
\hline 1173 & 0,219 & 0,219 & 1,028 & 2123 & 0,135 & 0,135 & 0,941 \\
\hline 1223 & 0,211 & 0,211 & 1,021 & 2173 & 0,132 & 0,132 & 0,938 \\
\hline 1273 & 0,204 & 0,204 & 1,015 & 2223 & 0,130 & 0,130 & 0,935 \\
\hline 1323 & 0,197 & 0,197 & 1,010 & 2273 & 0,128 & 0,128 & 0,932 \\
\hline 1373 & 0,191 & 0,191 & 1,004 & $T_{c r}=(3223)$ & - & $(0,101)$ & $(0,885)$ \\
\hline
\end{tabular}

The results of comparison of reference and calculated data on this characteristics are presented in Table 1.

Table 1. Reference [13] and Calculated (17) Values of Lithium Dynamic Viscosity and the Rate of a-Clusters Association. 
According to the Table the coordination of reference and culculated data are perfect which proves that the functional characteristic of the cluster and associate model (13) and its correspondence to physical nature of viscosity. The reduction of cluster association rate folloed by temperature increase proves the same; the closeness of a-values to 1 indicates the actual absence of the clusters' association which is typical for metal liquids [11].

Finally, gaseous state is analyzed in the framework of chaotic particles conception. Besides stated correlation between gases viscosity and the ratio of vapor-mobile particles which increase the viscosity by means of higher frequency of visco elastic encounters of the high-energy particles (high-velocity particles) [11], the direct dependency of the vaporization properties of solid and liquid matters on the ratio of particles which overcame the heat barrier of boiling, i.e. vapor-mobile particles [14]. The existed experimental data of saturated-vapour pressure of simple matters are analyzed on this basis and these data are presented in analytical temperature dependences in the whole range of existence of condensed state of these matters.

\section{Conclusion}

Thus, the heat energy, which is a measure of chaotic motion of particles and which is determined by Boltzmann distribution that is valid not only for gaseous but condensed state of matter [15], could be an analytical tool to reveal the united nature of solid, liquid and gaseous states based on introduction of new concepts of virtual crystal-mobile, liquid-mobile and vapor-mobile particles.

\section{References}

[1] E.M. Soroko. Structural Harmony of Systems. - Minsk: Science and Technology, 1984. -264 p.

[2] V.P. Malyshev. Probabilistic and Deterministic mapping. Almaty-Karaganda, Gylym, 1994. - 376 p.

[3] V.P. Malyshev, A.M. Nurmagambetova(Makasheva). The Concept of chaotic Particles as the Basis of a Single Display of Solid, Liquid and Gaseous States of Matter // Bulletin of the Kazakh National University, chemical Series. -2004. - № 3(35). -P. 53-67.
[4] V.P. Malyshev, A.M. Nurmagambetova. United interpretation of aggregate substance conditions by degree of its chaotization // Eurasian Physical technical journal. - 2004. - vol. 1. - № 2. -P. 10-14.

[5] V.P. Malyshev, N.S. Bekturganov, A.M. Nurmagambetova, T. Suleymenov. Development of a unified theory of chaotic particles for solid, liquid and gaseous states and its application to improve technology, increase production and improve the quality of blister copper and copper rods // Bulletin of the Kazakh National University, a series of chemical. - 2007. -№ 2(46). -P. 55-66.

[6] V.P. Malyshev, N.S. Bekturganov, A.M. Turdukozhayeva (Makasheva), T. Suleymenov. Basic concepts and according to the concept of randomized particles // Bulletin of the National Academy of Engineering. - 2009. - № 1. - P. 71-85.

[7] V.P. Malyshev, A.M. Turdukozhayeva, T. Suleymenov. Virtuality of solid, liquid and gaseous states of matter / / Encyclopedia chemical engineer. - 2009. - № 12. - P. 13-23.

[8] V.P. Malyshev, B.T. Abdrakhmanov, A.M. Nurmagambetova. Fusibility and plasticity of of metals. - Moscow: Scientific World, 2004. $-148 \mathrm{p}$.

[9] V.P. Malyshev, A.M. Makasheva. Relation self diffusion activation energy with the concept of randomized particles // Reports of the National Academy of Sciences of the Republic of Kazakhstan. - 2014. - № 2. - P. 15-21.

[10] V.P. Malyshev, A.M. Turdukozhayeva. Deformation and fracture of materials as a process of randomization // Deformation \& Fracture of Materials and Nanomaterials DFMN 2011 / Book of articles, ed. by O.A. Bannykhet. al. Moscow: InterkontaktNauka, 2011. - P. 73-74.

[11] V. Malyshev, A. Makasheva, N. Bekturganov. Viscosity, fluidity and density of the substances. Aspect of Chaotization. - Saarbücken: Lambert Academic Publishing, 2013. - 340 p.

[12] Turdukozhayeva A.M., Fedorovich Y.A., Malyshev V.P., Suleymenov T., Kazhikenova A.Sh. Cluster and Associate Model of Temperature Dependency of Liquid Borium Viscosity //Complex Application of Mineral Raw Materials. 2013. - No. 2. - P.68-74.

[13] Volkov A.I., Zharskiy I.M. Comprehensive Chemistry Reference Book. - Mn.: Sovremennaya Shkola, 2005. - 608 p.

[14] V.P. Malyshev, A.M. Turdukozhayeva, E.A. Ospanov, B. Sarkenov. Evaporation and boiling simple substances. Moscow: Scientific World, 2010.-304 p.

[15] M.A. Leontovich. Introduction to Thermodynamics. Statistical Physics. - Moscow: Higher school, 1983. - 416 p. 